\title{
High-resolution 3D shallow crustal structure in Long Beach, California: Application of ambient noise tomography on a dense seismic array
}

\author{
Fan-Chi Lin ${ }^{1}$, Dunzhu Li ${ }^{1}$, Robert W. Clayton ${ }^{1}$, and Dan Hollis ${ }^{2}$
}

\begin{abstract}
Ambient noise tomography has proven to be effective in resolving shallow earth structure. We applied ambient noise tomography on a dense seismic array in Long Beach, California. The array was composed of more than 5200 stations with an average spacing close to $100 \mathrm{~m}$. Three weeks of passive ambient noise were crosscorrelated between each station pair, which resulted in more than 13.5 million crosscorrelations within the area. Clear fundamental-mode Rayleigh waves were observed between 0.5 and $4 \mathrm{~Hz}$, which were most sensitive to structure above $1-\mathrm{km}$ depth. For each station pair, we applied frequency-time analysis to determine the phase traveltime dispersion, and, for each frequency, we applied eikonal tomography to determine the Rayleigh wave phase velocity map. The eikonal tomography accounted for ray bending by tracking the wavefront and allowed uncertainties to be estimated through statistical analysis. The compilation of phase velocity maps was then used to invert for 3D shear velocity structure. The inverted model showed clear correlation with the known geologic features such as the shallow south-north velocity dichotomy and a deeper fast anomaly associated with the NewportInglewood fault zone. Our results can potentially be used to complement traditional active source studies.
\end{abstract}

\section{INTRODUCTION}

Ambient noise tomography has been widely used to study shallow earth structure on regional (e.g., Sabra et al., 2005; Shapiro et al., 2005; Yao et al., 2006) and continental scales (e.g., Yang et al., 2007; Lin et al., 2008; Saygin and Kennett, 2012). The method starts by crosscorrelating passive ambient noise signals recorded by stations at different locations. The crosscorrelation between each station pair is then used to approximate the Green's function between the two locations (Bensen et al., 2007). In most applications, strong surface wave signals within the microseism frequency band $(0.05-0.2 \mathrm{~Hz})$ can be extracted and traditional surface wave tomography methods are then applied to invert for 3D shear velocity structure (e.g., Yao et al., 2008; Moschetti et al., 2010). Applications using ambient noise crosscorrelations to extract body wave signals are also beginning to emerge (e.g., Draganov et al., 2009; Zhan et al., 2010; Ruigrok et al., 2011; Poli et al., 2012a, 2012b; Lin et al., 2013).

Between January and June 2011, more than 5200 high-frequency (10-Hz corner frequency) velocity sensors were deployed in the Long Beach area as part of a petroleum industry survey (Figure 1). The survey area covers several segments of the active northwestsoutheast trending Newport-Inglewood fault system (Figure 1), which are also related to the petroleum reservoirs (Wright, 1991). Along with active signals emitted by high-frequency vibrator sources $(8-80 \mathrm{~Hz})$, passive ambient noise was also recorded continuously across the array. The availability of continuous passive noise records and the dense $2 \mathrm{D}$ station coverage provide a great opportunity to investigate detailed 3D shallow crustal structure in the Long Beach area based on ambient noise tomography.

In this study, we apply ambient noise tomography to the Long Beach array to demonstrate the applicability of the method to a dense short-period array. In contrast to typical ambient noise applications, the relative small aperture of the array prevents us from studying surface waves within the microseism frequency band, in which a single wavelength is comparable to or larger than the entire array. With the dense 2D array, however, we show here that high-frequency $(0.5-4 \mathrm{~Hz})$ Rayleigh waves can be extracted from noise crosscorrelations and used for high-resolution study of the $3 \mathrm{D}$ shear velocity structure above $1-\mathrm{km}$ depth. These high-frequency surface waves are typically difficult to observe for longer paths due to strong scattering and attenuation (Yang et al., 2011a). Note

\footnotetext{
Manuscript received by the Editor 26 October 2012; revised manuscript received 4 March 2013; published online 1 August 2013.

${ }^{1}$ California Institute of Technology, Division of Geological and Planetary Sciences, Seismological Laboratory, Pasadena, California, USA. E-mail: linf@ caltech.edu; dli@caltech.edu; clay@gps.caltech.edu.

${ }^{2}$ NodalSeismic LLC, Signal Hill, California, USA. E-mail: dan.hollis@nodalseismic.com.

(c) 2013 Society of Exploration Geophysicists. All rights reserved.
} 
that although the frequency range we studied is outside the nominal frequency band of the geophones (10-Hz corner frequency), strong Rayleigh wave signals are observed in the correlations.

For each frequency, we apply eikonal tomography (Lin et al., 2009; Gouédard et al., 2012) to determine the fundamental-mode Rayleigh-wave phase velocity map and its uncertainty. Eikonal tomography, which uses the array configuration to track the phase front empirically, accounts for ray bending and is theoretically more accurate than traditional straight ray inversions (e.g., Barmin et al., 2001). Because the method deals with traveltime measurements from the same common sources one source at a time, phase velocity and its uncertainty can be determined statistically based on the repeating measurements and the method is computationally very efficient. The 3D shear velocity inversion based on the Rayleigh wave phase velocity maps shows velocity anomalies that are correlated with known geologic features such as the shallow north-south velocity dichotomy and the slightly deeper fast anomaly associated with the Newport-Inglewood system.

\section{METHOD}

\section{Ambient noise crosscorrelation and virtual source}

Theoretical studies (e.g., Lobkis and Weaver, 2001; Snieder, 2004; Tsai, 2010) have shown that the crosscorrelation $\left(C_{A B}\right)$ of a diffuse, equipartitioned noise field at locations $A$ and $B$ is related to the Green's function between the two points $\left(G_{A B}\right.$ and $\left.G_{B A}\right)$ based on

$$
\frac{d C_{A B}(t)}{d t}=-G_{A B}(t)+G_{B A}(-t)-\infty<t<\infty .
$$

Although theoretically, $G_{A B}$ and $G_{B A}$ are identical due to the reciprocity principle, for an inhomogeneous noise source distribution, the negative and positive time lags of a crosscorrelation can be

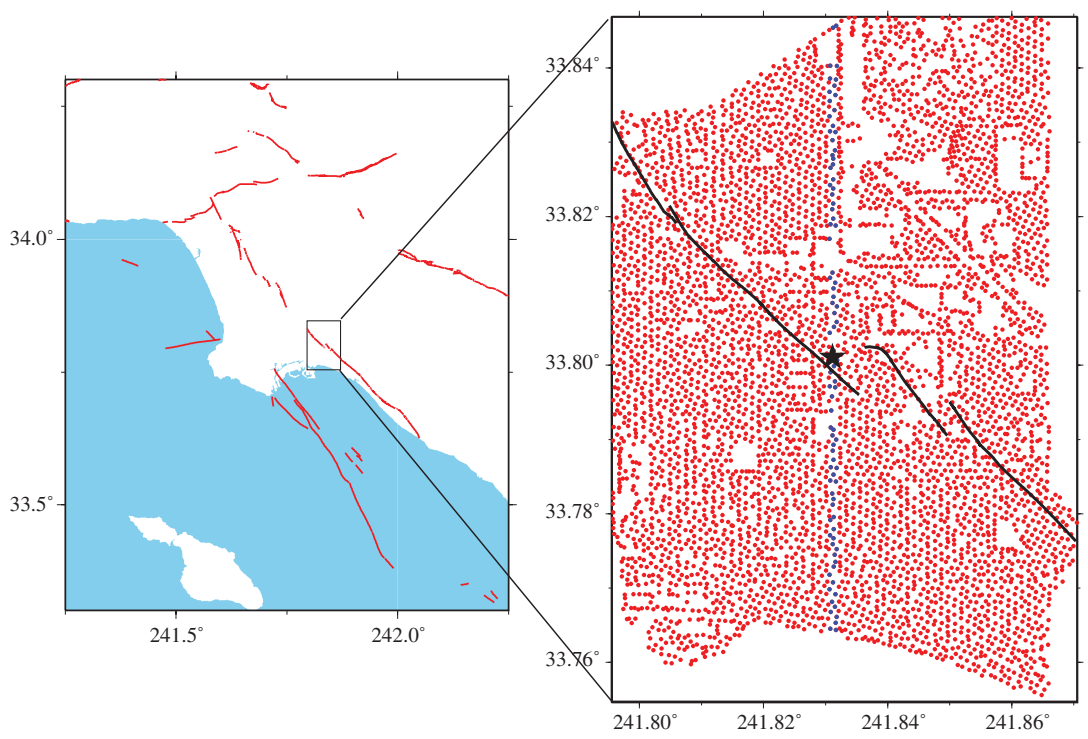

Figure 1. The array configuration and the regional fault lines in Southern California. The small circles show the 5204 stations used in this study. Several segments of the Newport-Inglewood fault system are denoted by black lines in the magnified plot. The star and blue circles show the stations used in Figure 2. different. In general, the negative and positive time lags correspond to noise signals traveling from $B$ to $A$ and $A$ to $B$, respectively.

We use the method described by Bensen et al. (2007) (modified slightly) to calculate the crosscorrelation for each station pair between 5 March and 25 March. With 5204 stations across the array, more than 13.5 million crosscorrelations $\left(\sim 5204^{2} / 2\right)$ are calculated. For each 1-h noise segment, we first perform spectrum whitening and then calculate the crosscorrelation. Unlike Bensen et al. (2007), we do not perform temporal normalization before crosscorrelation but normalize each 1-h noise crosscorrelation by its maximum amplitude (Prieto et al., 2011). As there are near 500 hourly crosscorrelation segments for each station pair, this process effectively suppresses the effects of irregular instrument spikes and large amplitude events. For each station pair, all normalized $1-\mathrm{h}$ crosscorrelations are then stacked together to get the final three-week crosscorrelation.

Figure 2 shows an example of the record section for the threeweek crosscorrelations between a common station near the center of the array and other stations on a north-south line (Figure 1). For low frequencies $(<1 \mathrm{~Hz}$; Figure 2b), clear fundamental-mode Rayleigh waves are observed in the negative and positive time lags for stations in the south and north, respectively. Note that weak higher mode/body wave signals, which propagate faster than fundamental-mode Rayleigh waves, are also observed. At higher frequencies (2-4 Hz; Figure 2c), the crosscorrelations are generally nosier, but a clear fundamental-mode Rayleigh wave can still be observed in the negative and positive time lags for stations in the south and north directions. Although understanding the detailed noise source distribution is not the main focus of this study, a visual inspection of noise crosscorrelations suggests that different noise sources between the low and high frequencies (Figure 2 and supplementary movies) are important. For low frequencies, the noise signals are dominantly propagating in the north and east directions, and hence they are likely to be oceanic related. For high frequencies, the propagating energy of the noise signals tends to be more isotropic and can either be generated by highly scattered oceanic sources or possibly anthropogenic activities.

To suppress the effect of an inhomogeneous source distribution and reduce measurement errors in phase velocity dispersion, we calculate the symmetric component of the crosscorrelation by averaging the positive and negative lag signals (Lin et al., 2008). For each station pair, this is equivalent to averaging the source distribution on both sides of the stations. Based on the relationship between crosscorrelation and the Green's function (equation 1), we use the symmetric-component crosscorrelations between one common station and all other stations to approximate the wavefield emitted by a virtual source at the common station location. An example of the constructed wavefield at two snapshots in time for a virtual source near the center of the array is shown in Figure 3. A clear fundamental-mode Rayleigh wave emitted by the virtual source can be observed in almost all directions. Note the discontinuous wavefront in the southeastern direction near the Newport-Inglewood fault zone, which suggests that the fault zone acts as a velocity structure boundary. 
a)

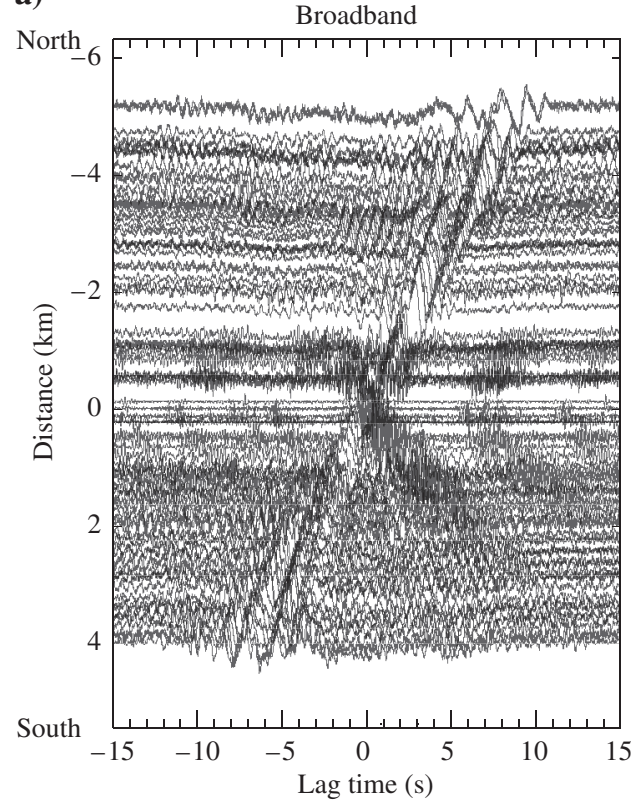

b)

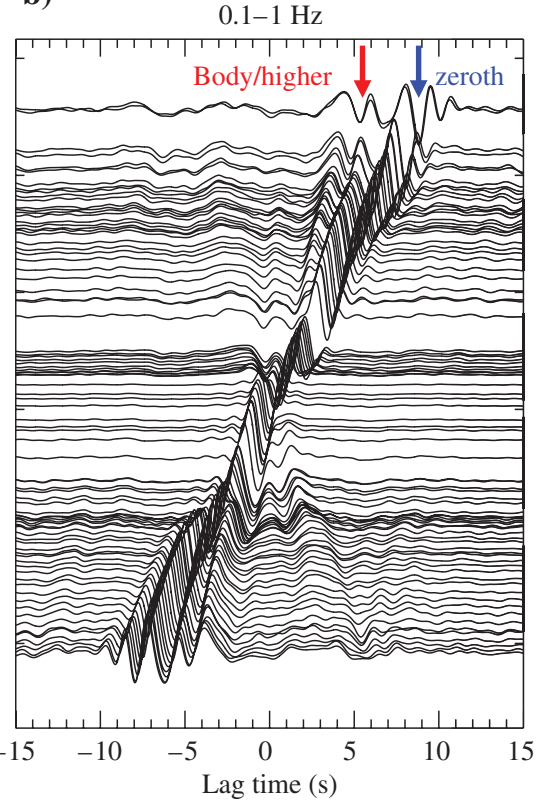

c)

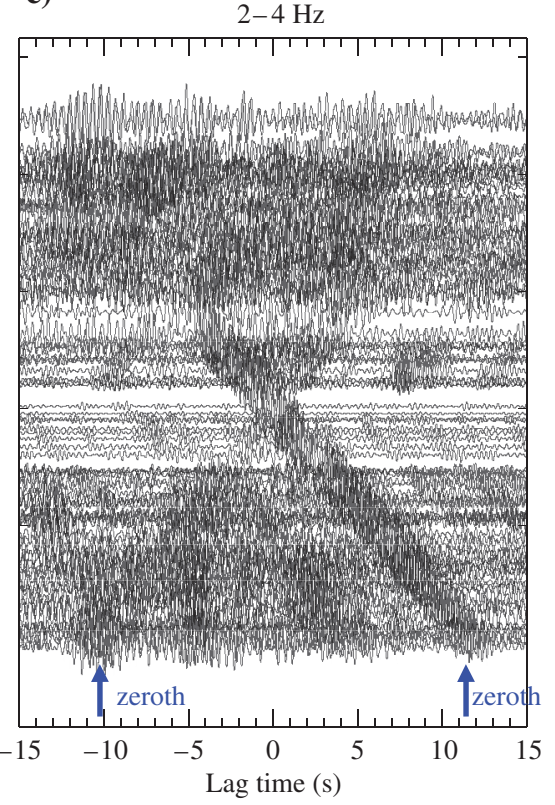

Figure 2. The record section of crosscorrelations calculated between a center station (star in Figure 1) and stations on a north-south line (blue circles in Figure 1). The crosscorrelations are sorted based on the path distances, in which we assign a negative sign if the second station is in northern direction compared to the common station. (a-c) Results without band-passed, $0.1-1 \mathrm{~Hz}$ band-passed, and 2-4 Hz band-passed signals, respectively. The fundamental Rayleigh wave signals are indicated by blue arrows in (b) and (c), where body wave/higher mode signals are indicated by red arrows in (b). Supplementary video clips are available online: S1, 10.1190/geo2012-0453.2; S2, 10.1190/ geo2012-0453.3; and S3, 10.1190/geo2012-0453.4. (S1) The wavefield constructed based on the ambient noise crosscorrelations between a common station and all other stations. The common station is located at the center of the equidistant contours, which are separated by $1 \mathrm{~km}$ intervals. For each station location and each snapshot in time, we assign the color based on the observed crosscorrelation waveform. The movie is running between -8 and $8 \mathrm{~s}$ lag time, where the last number in the lower left corner infers the time. Note that the negative and positive components of crosscorrelations are not stacked, which retains the original directionality of the noise wavefield. (S2) Same as S1, but bandpassed between 0.2 and $1 \mathrm{~Hz}$. The movie is running between -12 and $12 \mathrm{~s} \mathrm{lag} \mathrm{time.} \mathrm{(S3)} \mathrm{Same} \mathrm{as} \mathrm{S1} \mathrm{but} \mathrm{band-passed} \mathrm{between} 2$ and $4 \mathrm{~Hz}$. The movie is running between -8 and $8 \mathrm{~s}$ lag time.

a)

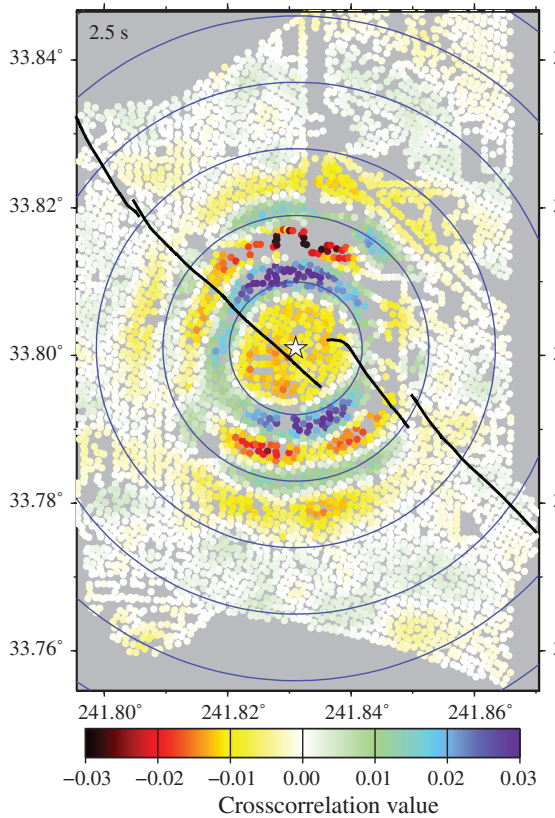

b)

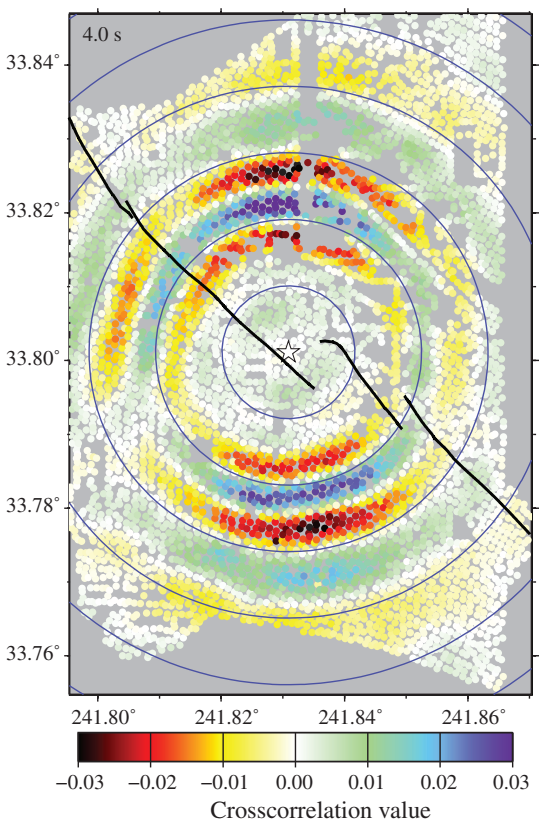

Figure 3. The wavefield emitted by a virtual source. The location of the virtual source is shown by the star near the center of the array. (a, b) Snapshots of the $0.5-1 \mathrm{~Hz}$ band-passed wavefield observed at each station location at 2.5- and 4.0-s lag times, respectively. The source distance contours are separated by $1-\mathrm{km}$ intervals. 
Caution must be exercised when relating the crosscorrelation and the Green's function based on equation 1 (Yao and van der Hilst, 2009), particularly for the amplitude response. The strengths of the observed surface wave signals are clearly directionally dependent (Figure 3). The noise source distribution, although likely smoothly varying spatially, is not homogeneous and hence does not fully satisfy the basic assumption of equation 1 . It is important to note that the wavefield emitted by a virtual source constructed by the ambient noise crosscorrelations (i.e., Figure 3) satisfies the governing wave equation. This allows the phase velocity structure to be determined by directly studying local wave propagation using eikonal tomography without the exact knowledge of the noise source distribution. To see this, consider any wavefield $u$ satisfying the homogeneous wave equation:

$$
\mathcal{L} u(r, t)=0,
$$

where $\mathcal{L}$ represents the linear differential operator. The time domain crosscorrelation, $C_{A}(\mathbf{r}, t)$, between one common location $\mathbf{r}_{A}$ and all other locations $\mathbf{r}$ of the wavefield $u$, also satisfies the wave equation as

$$
\begin{aligned}
\mathcal{L} C_{A}(r, t) & =\mathcal{L} \int u\left(r_{A}, \tau\right) u(r, \tau+t) d \tau \\
& =\int u\left(r_{A}, \tau\right) \mathcal{L} u(r, \tau+t) d \tau=0
\end{aligned}
$$

If we now consider $u$ to be the noise wavefield, which is dominantly the superposition of many ballistic waves, then the noise cross- correlation $C_{A}(\mathbf{r}, t)$ between one common location $\mathbf{r}_{A}$ and all other locations $\mathbf{r}$ will also satisfy the wave equation. If we neglect attenuation, this implies that the wavefield emitted by a virtual source at $\mathbf{r}_{A}$ constructed by stacking positive and negative component crosscorrelations $\left[C_{A}(\mathbf{r},-t)+C_{A}(\mathbf{r}, t)\right] / 2$ will also satisfy the wave equation. As we will discuss later, this allows eikonal tomography to be applied even with an inhomogeneous noise source distribution as long as a well-defined wavefront can be identified for the wavefield emitted by a virtual source.

\section{Eikonal tomography}

We closely follow the eikonal tomography method described by Lin et al. (2009) to determine Rayleigh wave phase velocity maps between the $0.5-$ and $4-\mathrm{Hz}$ frequencies. For each frequency, we measure traveltime between each station pair based on frequency-time analysis (Bensen et al., 2007; Lin et al., 2008). We assume that the fundamental Rayleigh wave is the dominating signal within the prediction window, which is defined by assuming a group velocity between 0.3 and $1.5 \mathrm{~km} / \mathrm{s}$. The potential contamination from higher mode and body wave signals (Figure 2) can introduce errors in the traveltime measurement, and the errors will contribute to the uncertainty of our velocity estimation. Only measurements with a frequency-dependent signal-to-noise ratio $(\mathrm{S} / \mathrm{N})$ higher than eight are kept for further analysis. Here we define the $\mathrm{S} / \mathrm{N}$ by the ratio between the peak amplitude within the prediction window and the rms amplitude of the noise outside of the prediction window. a)

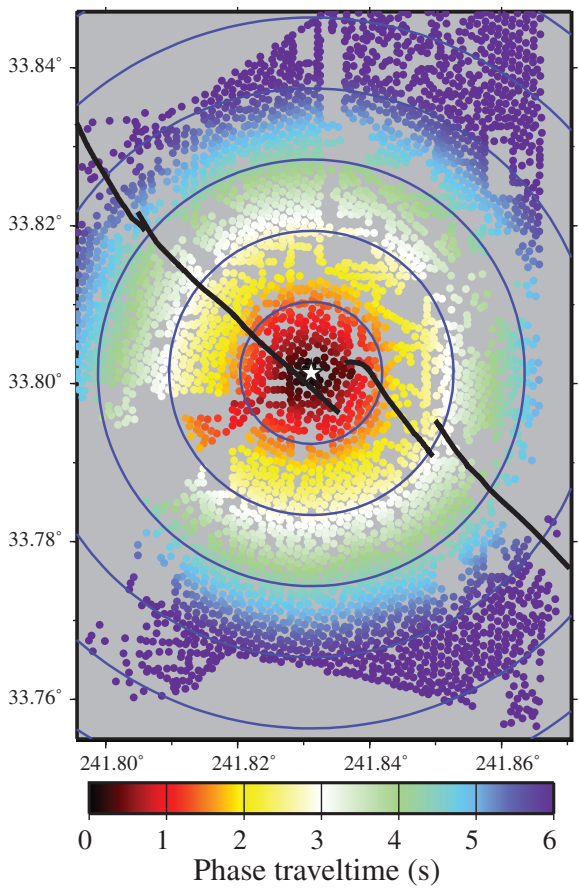

b)

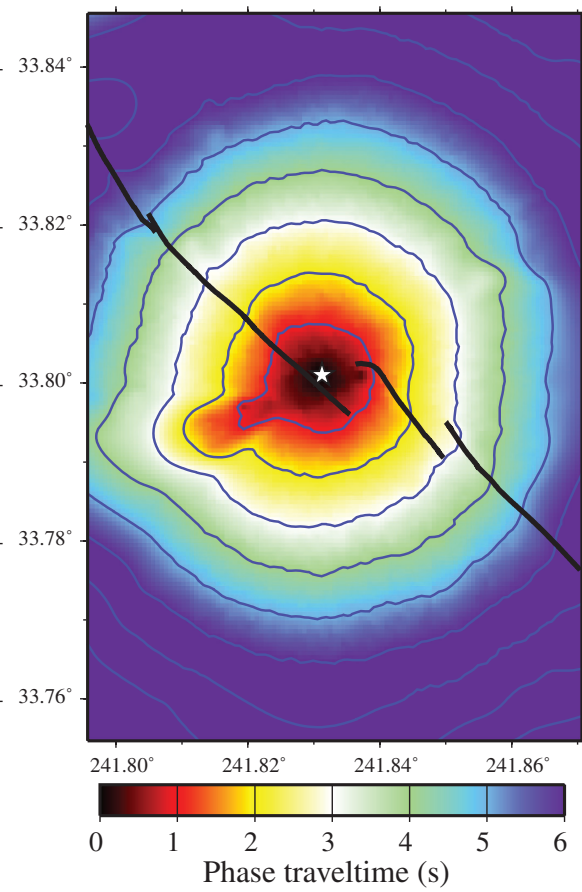

c)

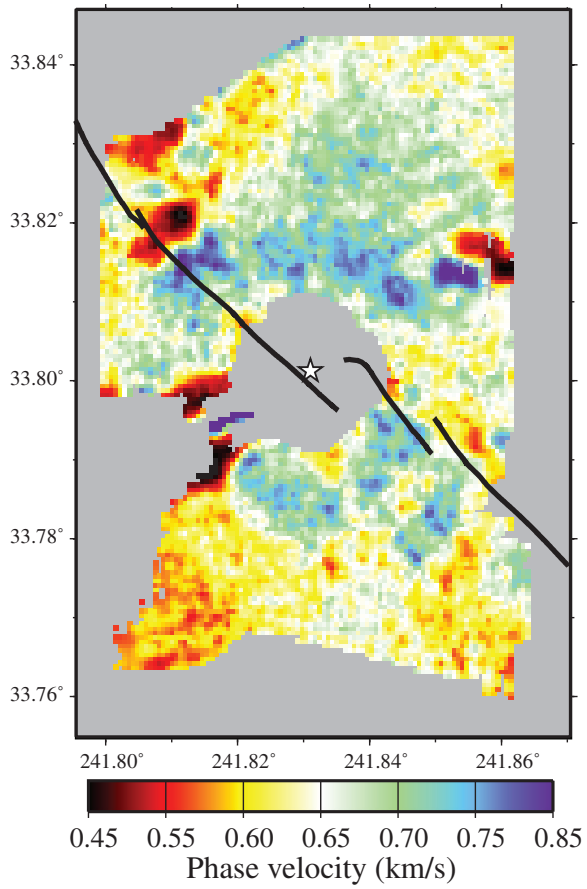

Figure 4. A demonstration of eikonal tomography. (a) The 1-Hz Rayleigh wave phase traveltime observed across the array for the wavefield shown in Figure 3. Only stations with S/N higher than our selection criterion are shown. The source distance contours are separated by $1-\mathrm{km}$ interval. (b) The phase traveltime map derived from (a) using the minimum curvature fitting method. The traveltime contours are separated by a 1-s interval. (c) The phase velocity map derived from (b) based on the eikonal equation (equation 4). Only areas satisfying our one-period traveltime and three- out of four-quadrant selection criteria are shown. 
Eikonal tomography deals with all measurements from the same virtual source simultaneously. An example of the $1-\mathrm{Hz}$ traveltime measurements for the wavefield shown in Figure 3 is shown in Figure $4 \mathrm{a}$. Note that the wavefield in the southwestern direction of the virtual source is generally weaker (Figure 3), and many measurements in that direction have an $\mathrm{S} / \mathrm{N}$ smaller than the selection criterion, and hence they are removed from further analysis (Figure 4a). Following Lin et al. (2009), all available traveltime measurements are used to construct the phase traveltime maps on a $60 \times 60 \mathrm{~m}$ grid based on minimum curvature fitting (Figure $4 \mathrm{~b}$; Smith and Wessel, 1990). The gradient of the traveltime map at each location is then used to estimate the apparent phase velocity (Figure $4 \mathrm{c}$ ) and the direction of wave propagation based on the eikonal equation

$$
\frac{\hat{k}}{c}=\nabla \tau,
$$

where $\hat{k}$ denotes the direction of wave propagation, $c$ is the apparent phase velocity, and $\tau$ is the phase traveltime.

The eikonal equation is derived directly from 2D wave equation using high-frequency approximation (Wielandt, 1993; Lin and Ritzwoller, 2011a). Because the wavefield emitted by a virtual source has a well-defined wavefront (e.g., Figure 3) and satisfies the wave equation (as discussed previously), the phase velocity determined based on equation 4 is likely not sensitive to the exact noise source distribution. Although an inhomogeneous noise source distribution can introduce traveltime biases when relating the crosscorrelations and Green's functions based on equation 1 (Yao and van der Hilst, 2009), the biases tend to be similar for nearby stations and cancel out when the spatial derivative in equation 4 is taken.

For example, if we consider an extreme case in which for an isotropic medium all noises are coming from the south, then because the positive and negative components of crosscorrelations are averaged, the wavefield emitted by a virtual source near the center of the array will be two plane waves, one propagating northward and one propagating southward. The traveltime variation will be zero on the east-west line through the virtual source location but increase monotonically toward north and south directions. The traveltime map will clearly be different from that expected for a point source, but the 2D gradient of the map does correctly reflect the direction of wave propagation (propagating northward and southward in the north and south, respectively) and phase velocity based on equation 4 everywhere but the zero traveltime line. On that line, the gradient cannot be determined due to the singularity, and our method will remove the region near the singularity by imposing a traveltime selection criterion as discussed below. Note that applying a traditional straight ray tomography method (e.g., Barmin et al., 2001) would introduce artificial azimuthal anisotropy in this case due to the uneven noise source distribution.

To retain only the most reliable measurements and avoid singularities, we remove regions with phase traveltime smaller than one period and only keep the regions where at least three of the four quadrants of the east-west and north-south axes are occupied by at least one station closer than $400 \mathrm{~m}$ (Lin et al., 2009). The one-period traveltime criterion is less restrictive than the more common recommendations of two to three periods used in regional studies (e.g., Lin et al., 2008; Yao et al., 2011), but due to the small aperture of the array, using a larger traveltime criterion, such as 2 periods, will degrade our ability to determine phase velocity maps at lower frequencies $(<0.7 \mathrm{~Hz})$. At higher frequencies $(>1.0 \mathrm{~Hz})$, using one or two periods of traveltime criterion results in very similar phase velocity maps, and the overall difference between the two is consistent with our error estimation. Note that there are two reasons to discard short-distance measurements in traditional ambient noise tomography applications. The first is to satisfy the far-field approximation, which mostly affects measurements within one wavelength, and the second reason is to remediate inhomogeneous noise source distributions, which can affect measurements at longer distances (Yao and van der Hilst, 2009). As we described, eikonal tomography is less sensitive to the noises source distribution, which somewhat justifies the shorter traveltime selection criterion used here.

The phase velocity map determined by a single virtual source (e.g., Figure 4c) is statistically unreliable, but an average over all measurements from different virtual sources reduces the uncertainty significantly. For each location, we calculate the mean and the standard deviation of the mean of all available measurements to determine the final phase velocity and its uncertainty, respectively. Figure 5 shows the result of $1-\mathrm{Hz}$ phase velocity maps and their associated uncertainty estimation when different numbers of virtual sources are used. Although the large-scale patterns of phase velocity maps are generally similar, the detailed features become more coherent and the overall uncertainty is reduced when more virtual sources are used. Note that the uncertainty estimated here accounts for not only random measurement variations but also systematic measurement variations (e.g., due to azimuthal anisotropy for waves propagating in different directions). The final phase velocity maps at 2 and $0.67 \mathrm{~Hz}$ with all virtual sources used are summarized in Figure 6. The 0.67-, 1-, and 2-Hz Rayleigh waves shown in Figures 5 and 6 are most sensitive to structure near 400, 250, and $100 \mathrm{~m}$, respectively. The quality of the map degrades at the lower frequencies due to fewer measurements satisfying the oneperiod traveltime selection criterion and potential errors due to finite frequency effects (Lin and Ritzwoller, 2011a).

\section{Three-dimensional inversion}

Based on phase velocity maps observed between the 0.5 - and $4-\mathrm{Hz}$ frequencies, we invert for the top $1-\mathrm{km} 3 \mathrm{D}$ shear velocity structure in the Long Beach area. We smooth the phase velocity maps by a 500-m radius Gaussian filter before the inversion to ensure the smoothness of the inverted model. For each location, the Rayleigh wave phase velocity dispersion curve and its associated uncertainty are used to invert for 1D shear velocity structure beneath that location. The dispersion measurements at two example locations (Figure 6a) are shown in Figure 7a. Faster dispersion measurements are clearly observed for the point close to the Newport-Inglewood fault zone compared to the point in the southwest. Note that the uncertainties of the dispersion measurements are higher near lower and higher frequency ends. At the lower frequency end, this is mostly due to fewer measurements satisfying the oneperiod traveltime criterion and measurement errors due to unaccounted finite frequency effects. At the higher frequency end, this is primarily due to the smaller $\mathrm{S} / \mathrm{N}$ of the Rayleigh wave signals, which is likely due to strong scattering and attenuation and the lower convergence rate of the crosscorrelation to the Green's function.

Because Rayleigh wave dispersion measurements have considerably broad depth sensitivity kernels and do not have the ability to resolve velocity discontinuities, we parameterize our $1 \mathrm{D}$ model at 
each location by five cubic B-splines as shown in Figure $8 \mathrm{a}$. In the inversion, we fix the $V_{\mathrm{P}} / V_{\mathrm{S}}$ ratio and the density models (Figure $8 \mathrm{~b}$ ) and only perturb the shear velocity model. We minimize the misfit between observed and predicted dispersion curves (Herrmann and Ammon, 2002) through a steepest descent nonlinear inversion method (Lin et al., 2012). A half-space model beneath 1-km depth is used. Tests show that small changes in the $V_{\mathrm{P}} / V_{\mathrm{S}}$ ratio and density models do not change the inverted shear velocity model significantly. Figure $7 \mathrm{~b}$ shows the inverted shear velocity models for the two sample locations, and the predicted dispersion curves are shown in Figure $7 \mathrm{a}$.

\section{RESULTS AND DISCUSSION}

\section{$3 D$ model}

The compilation of all the inverted 1D models at different locations is used to construct the $3 \mathrm{D}$ model. The shear velocity model at three different depths is shown in Figure 9, where the average 1D model for the whole area is shown in Figure 10a. In general, at 100-, 300-, and 650-m depths, the model shows very similar velocity patterns as observed in Rayleigh wave phase velocity maps at 2, 1, and $0.67 \mathrm{~Hz}$, respectively (Figures 5 and 6). This is expected considering the depth sensitivity kernels of the Rayleigh waves (Figure 10b).

At shallow depths (<100 m; e.g., Figure 9a), a clear north-south dichotomy with the transition near the Newport-Inglewood fault is observed. The velocity difference, slow in the south and fast in the north, can be due to the difference in geologic history and the aquifer system separated by the fault zone. The NewportInglewood fault zone is a right-lateral transpressional system, which manifests itself as a line of hills on the earth surface and is a natural geologic boundary in the area (Wright, 1991; Wesnousky, 2005). At greater depths (>200 m; e.g., Figure 9b and 9c), a fast anomaly associated with the Newport-Inglewood fault system starts to emerge likely related to deeper earth material exhumed due to the deformation process. Figure 11 shows three north-south cross sections of the model. A clear north-south dichotomy in the top $100 \mathrm{~m}$ is again evident. Spatial variations of the fault-related fast
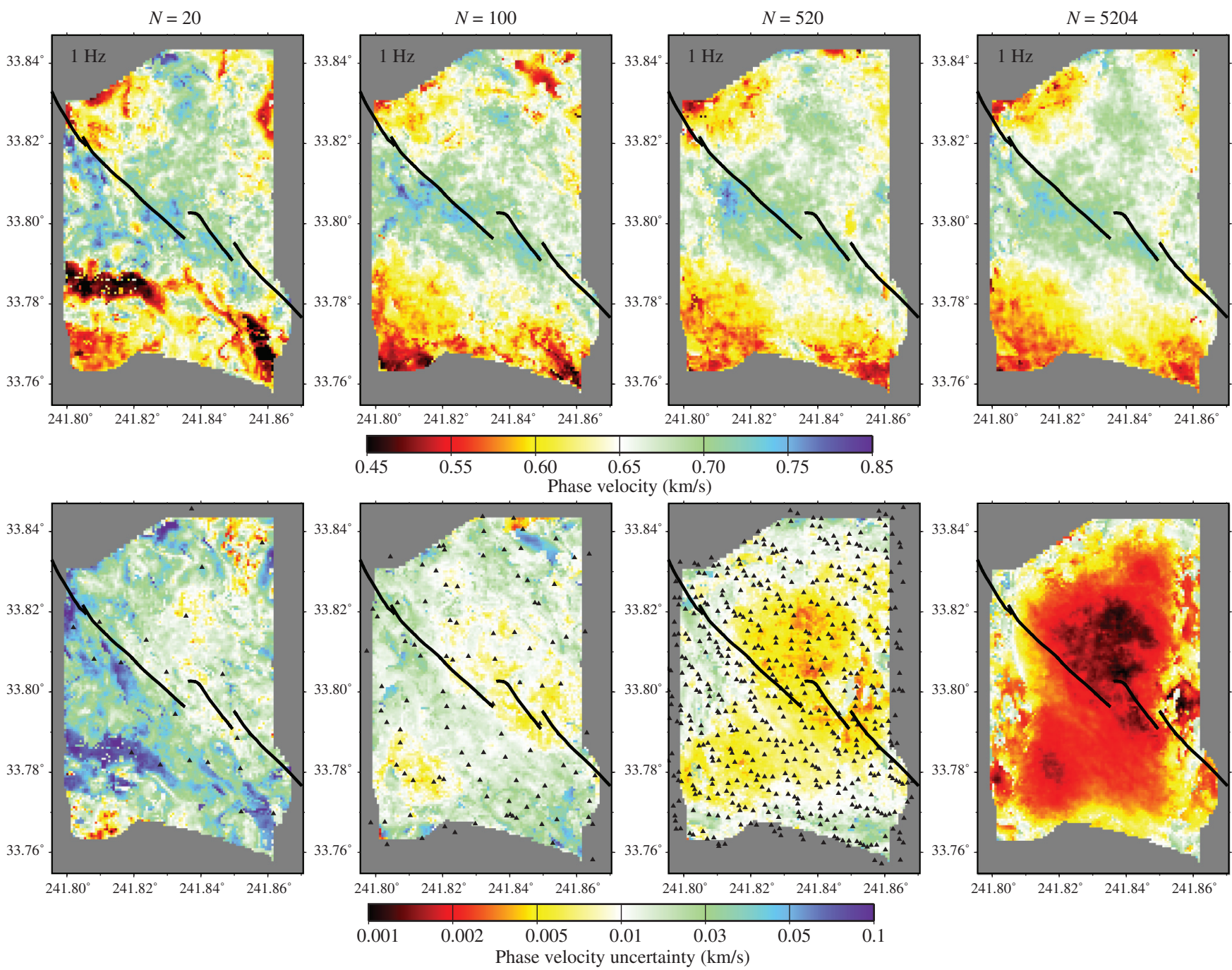

Figure 5. The 1-Hz Rayleigh wave phase velocity map (upper) and its associated uncertainty estimation (lower) based on different numbers of virtual sources. The number of virtual sources in each plot is shown on the top. Besides $N$ equals to 5204, where all stations are used, the triangles in the lower plots show the virtual source locations used. 
anomaly is also observed, where the fast anomaly in cross sections B-B ${ }^{\prime}$ and $\mathrm{C}-\mathrm{C}^{\prime}$, which cut through the high topography hills (i.e., Signal Hill) in the region, plunges to the southeast.

The ability to construct a high-resolution shallow shear velocity model has several important applications. First, the model provides complementary information to the traditional exploration seismology analysis, which is based on active sources and focuses on $\mathrm{P}$-wave reflection imaging. The availability of a shallow 3D velocity model will allow static correction to be estimated as the vertical traveltime through the model. Generating the model with surface waves means that there does not need to be a coherent refractor or reflector to estimate these corrections. Second, the model provides new ways to investigate earthquake hazard assessment. The shear velocity model is particularly important to predict strong ground motion during a seismic event. Third, the model provides new insights to the active fault zone structure with an unprecedented resolution.

\section{Azimuthal anisotropy}

Along with the isotropic structure, eikonal tomography also straightforwardly allows azimuthal anisotropy and its uncertainty to be evaluated (Lin et al., 2009, 2011). Azimuthal anisotropy describes the velocity difference for waves propagating in different directions and in the very shallow earth is likely due to the alignment of microcracks (Crampin, 1994). Because such alignment is mostly stress driven, the ability to determine azimuthal anisotropy at the shallow depth can have potentially important implications on the ability to determine the regional stress field.

Based on the eikonal equation (equation 4), for each phase velocity measurement, we approximate the direction of wave propagation by the direction of the traveltime gradient. For each frequency and location, we follow the method described by Lin et al. (2009) to statistically summarize all available phase velocity measurements within each $20^{\circ}$ azimuth bin (Figure 12a-12c). The resulting directionally dependent phase velocity measurements and their uncertainties are then used to determine the anisotropy parameters. For weakly anisotropic media (Smith and Dahlen, 1973), the Rayleigh wave phase velocity $c$ can be expressed as

$$
\begin{aligned}
c(\psi)= & c 0+A \cos [2(\psi-\varphi)] \\
& +B \cos [4(\psi-\alpha)],
\end{aligned}
$$

where $\psi$ is the azimuthal angle, $c 0$ is the isotropic velocity, $A$ and $B$ are the amplitude of anisotropy, and $\varphi$ and $\alpha$ define the orientation of the anisotropic fast axes for the $2 \psi$ and $4 \psi$ components of anisotropy. In many applications, $2 \psi$ is the dominant anisotropy component observed when the finite frequency effect is not severe (e.g., Figure 12a-12c; Lin et al., 2011; Lin and Ritzwoller, 2011b). Here, we fit the directionally dependent phase velocity based on equation 5 without the $4 \psi$ component.

The azimuthal anisotropy fast directions and amplitudes for 1.25and $1.0-\mathrm{Hz}$ Rayleigh waves, which are most sensitive to structure near the 150- and 200-m depths (Figure 10b), are summarized in Figure $12 \mathrm{~d}$ and $12 \mathrm{e}$, respectively. Note that the isotropic velocities $c 0$ determined here are very similar to those determined earlier by a)

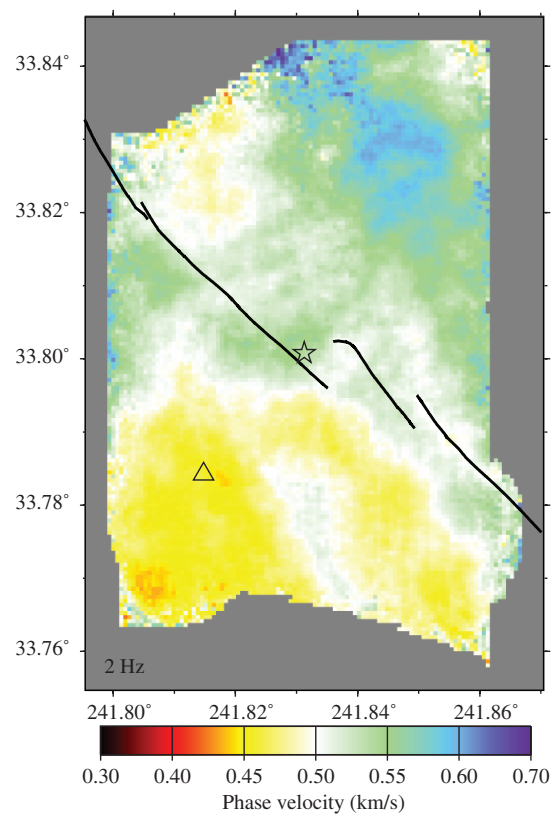

b)

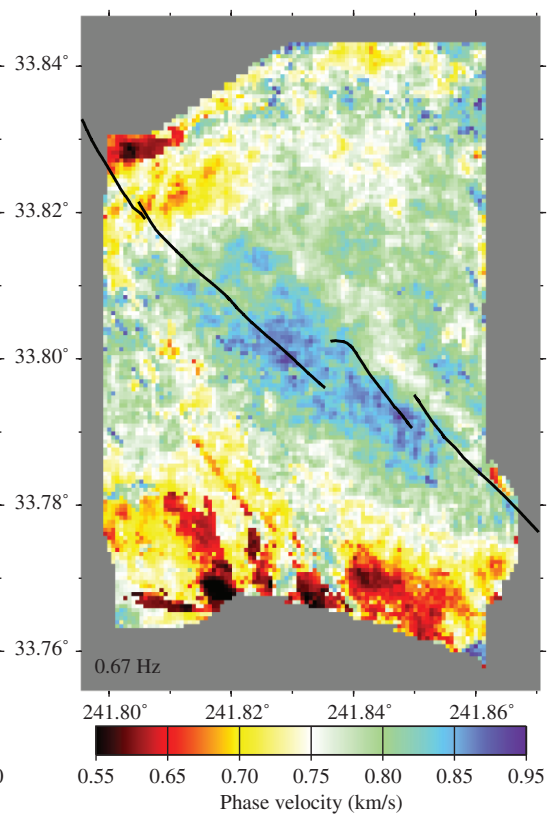

Figure 6. $(\mathrm{a}, \mathrm{b})$ The 2- and $0.67-\mathrm{Hz}$ Rayleigh wave phase velocity maps. The star and triangle in (a) denote the locations used in Figure 7. a)

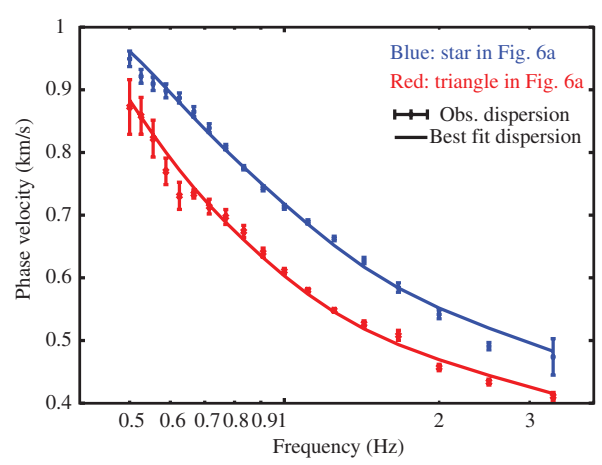

b)

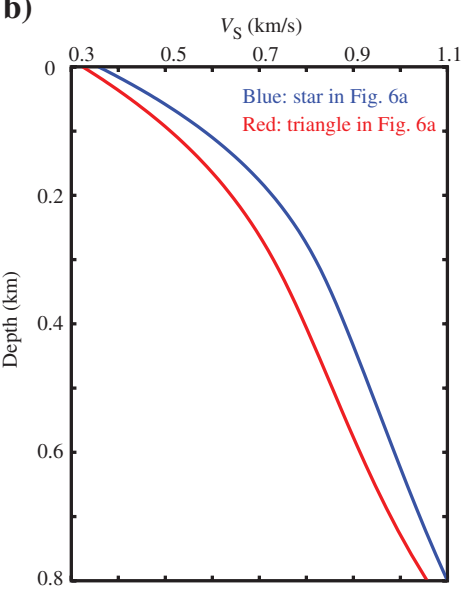

Figure 7. (a) Rayleigh wave dispersions at two locations shown in Figure 6a. The dispersion measurements and their uncertainties are taken from the phase velocity maps derived by eikonal tomography. The solid lines are the predicted dispersion curves based on the inverted 1D model shown in (b). (b) Inverted 1D model for dispersion measurements shown in (a). 
taking the mean of all measurements. The anisotropy fast directions are overall oriented north-south, which is consistent with the maximum horizontal compressional stress direction in Southern California (Heidbach et al., 2008; Yang et al., 2011b) and suggests that the anisotropy is related to the regional stress field. Small-scale variation is also observed near the segmentation of the fault zone, which suggests either a small-scale stress change near the fault zone segmentation or the presence of fault parallel structural anisotropy (e.g., de Lorenzo and Trabace, 2011). Fast directions rotating toward the fault direction, in particular, can be clearly observed within the fault segment near Signal Hill at $1.0 \mathrm{~Hz}$. The uncertainties are generally higher at the lower frequency potentially due to the finite
Figure 8. (a) Five cubic B-splines used for describing our 1D model. Note that the shallow $\mathrm{B}$-splines are chosen to be slightly narrower to allow large velocity variation near the surface. (b) The $1 \mathrm{D}$ reference $V_{\mathrm{P}} / V_{\mathrm{S}}$ ratio and density models. a)

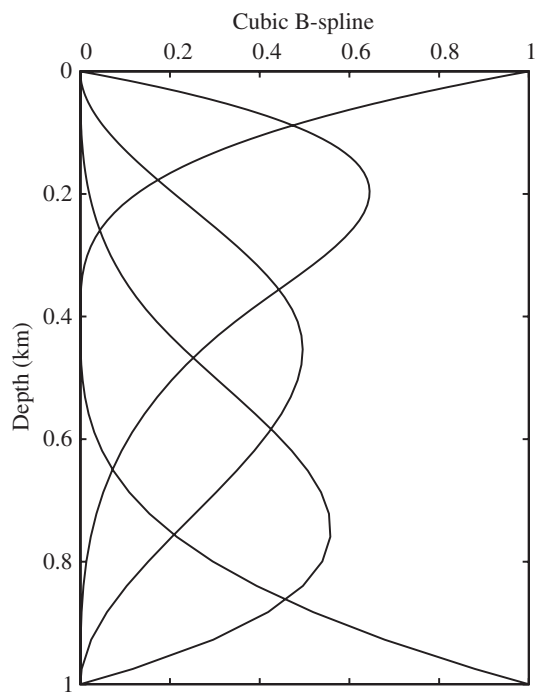

b)

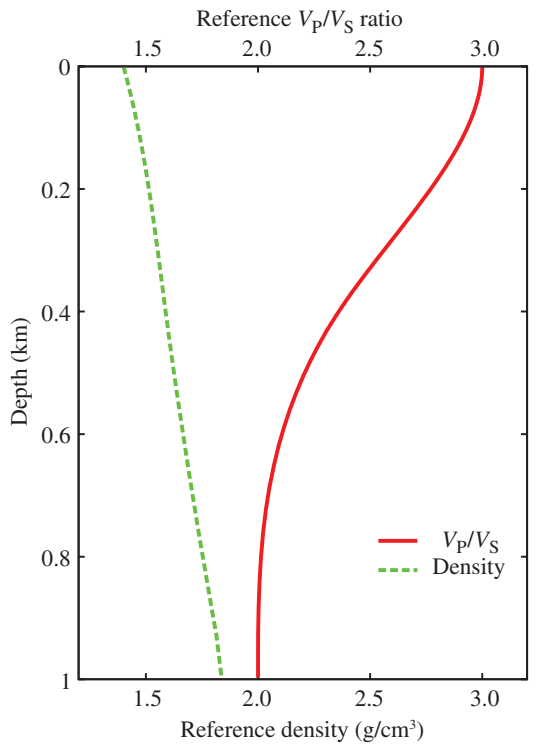

a)

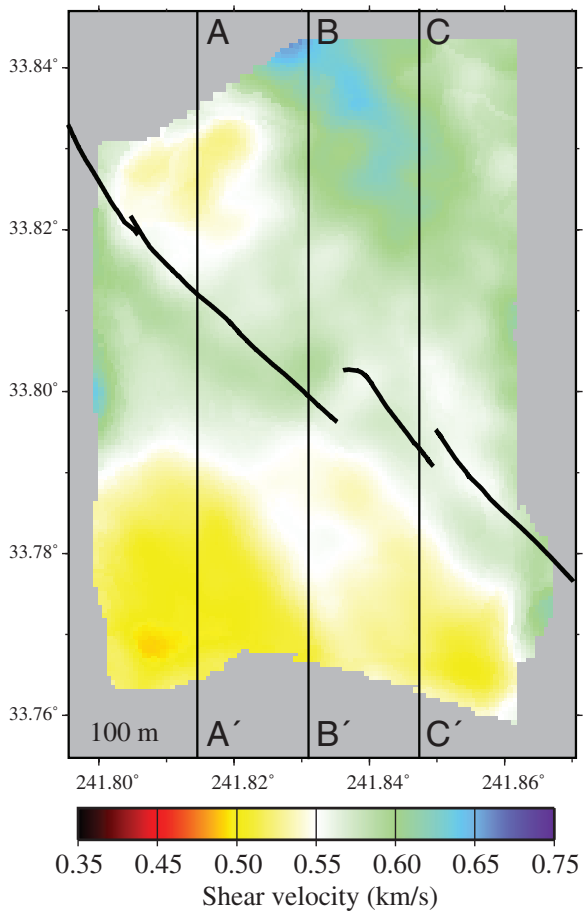

b)

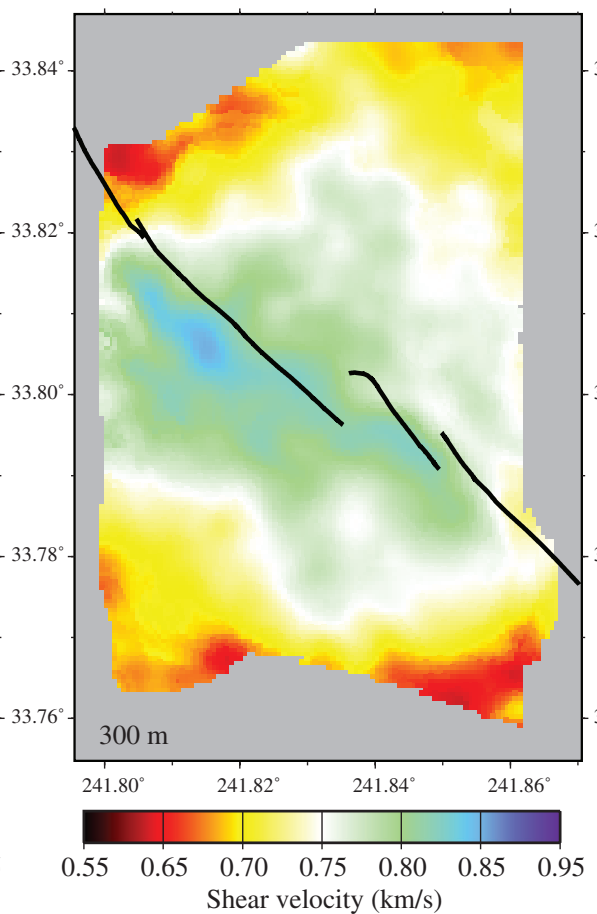

c)

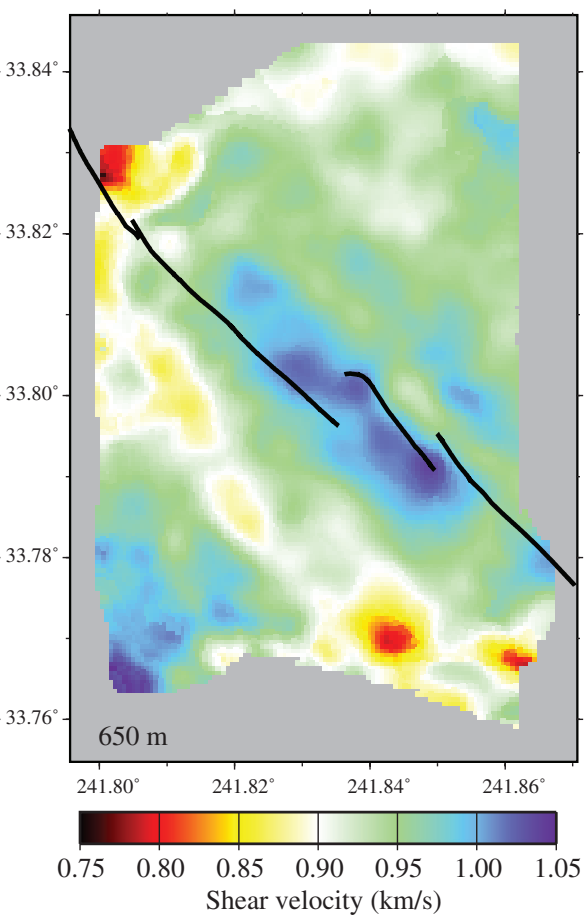

Figure 9. (a-c) Inverted 3D shear velocity model at 100-, 300-, and 650-m depths. The three north-south cross sections used in Figure 11 are shown in (a). 
frequency effect contamination (Lin and Ritzwoller, 2011b). In most locations, the anisotropy amplitudes are comparable or larger than $10 \%$, consistent with earlier shallow sedimentary studies in the Southern California region (Crampin, 1994; Coutant, 1996). The inversion of the 3D azimuthal anisotropy structure is out of the scope of this study and will be the subject of future contributions.

\section{Body wave and higher modes}

Although the theory (equation 1) suggests that the entire Green's function can be derived from the noise crosscorrelation for a diffuse noise field, in reality, the noise sources are distributed generally near the surface and hence body wave phases are not easily observed. However, extracting body waves from ambient noise is important for imaging subsurface structure without artificial sources.

Although fundamental Rayleigh waves are the dominant signals observed in our noise crosscorrelations, weak body wave/higher mode signals are also observed (Figure 2). Body waves and higher modes are sometime difficult to distinguish for short paths, particularly at low frequencies. To better demonstrate the body waves/higher modes, we stack all crosscorrelations within each 200-m distance bin (Figure 13). Because body waves/higher modes are more sensitive to deeper structure compared with fundamental Rayleigh waves, they tend to stack more coherently due to a smaller velocity variation. Clear body waves/higher models can be observed for low and high frequencies. Between 2 and $4 \mathrm{~Hz}$, a clear P-wave signal emerges with an $\sim 2-\mathrm{km} / \mathrm{s}$ apparent velocity. a)

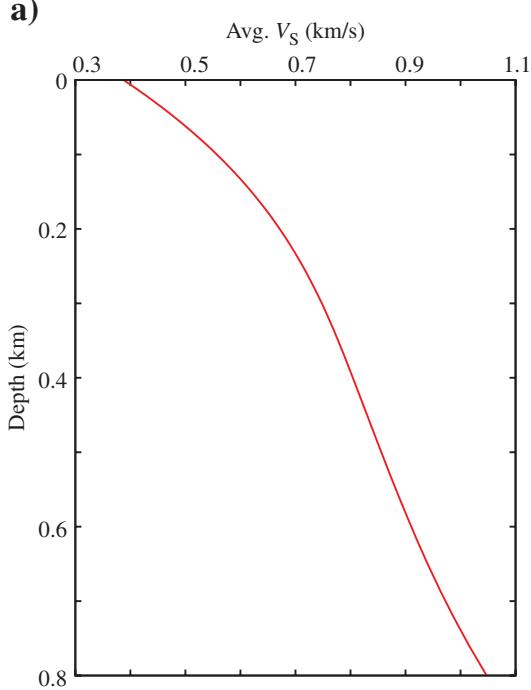

b)

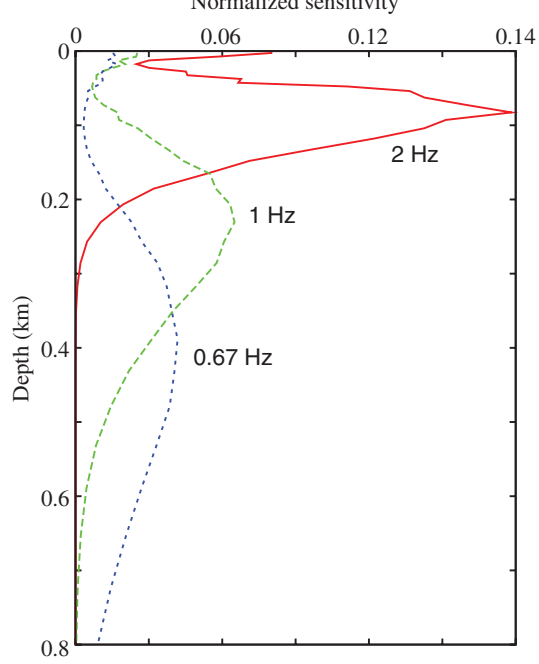

Figure 10. (a) Average 1D shear velocity model. (b) Depth sensitivity kernels for 0.67-, 1-, and 2$\mathrm{Hz}$ Rayleigh waves calculated based on the 1D model shown in (a).

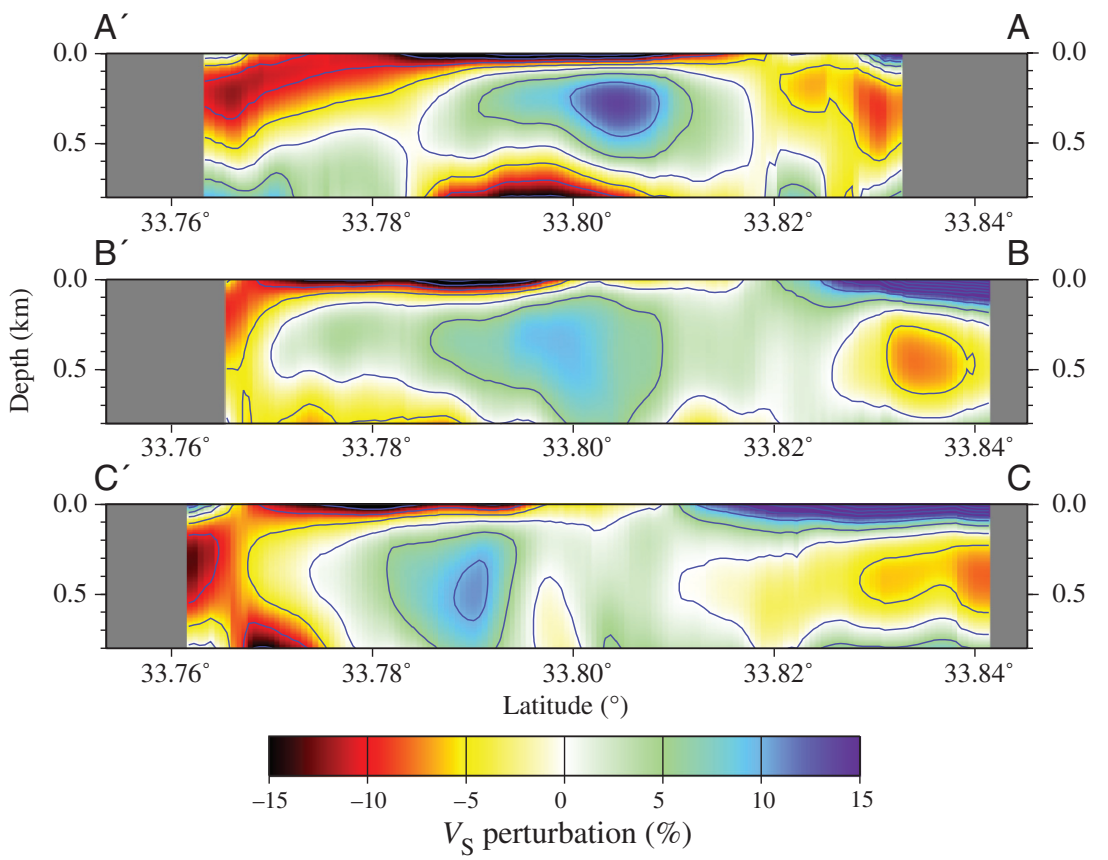

Figure 11. The three cross sections (Figure 9a) of the inverted 3D shear velocity model. Here, the average 1D model shown in Figure 10a is used as the reference and only the relative perturbation at each depth is plotted. The contours are separated by $5 \% V_{\mathrm{S}}$ velocity perturbation. 


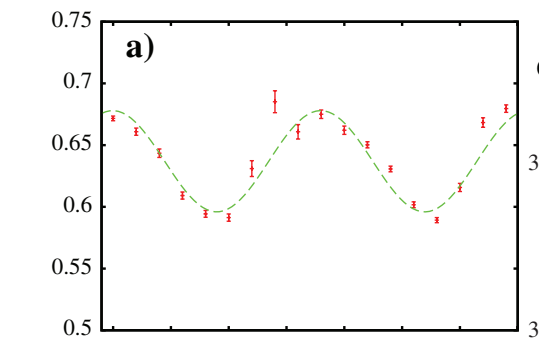

d)
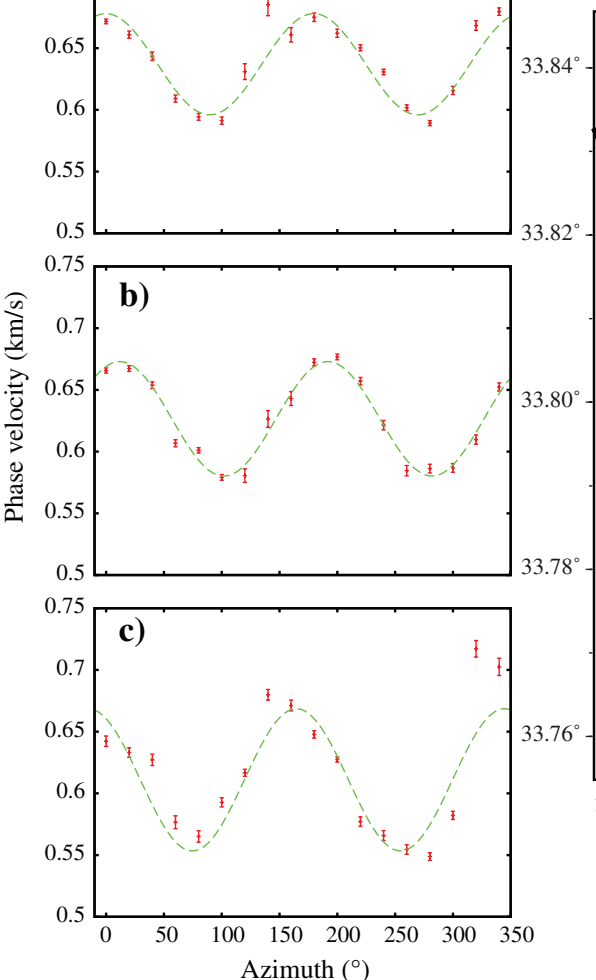

e)

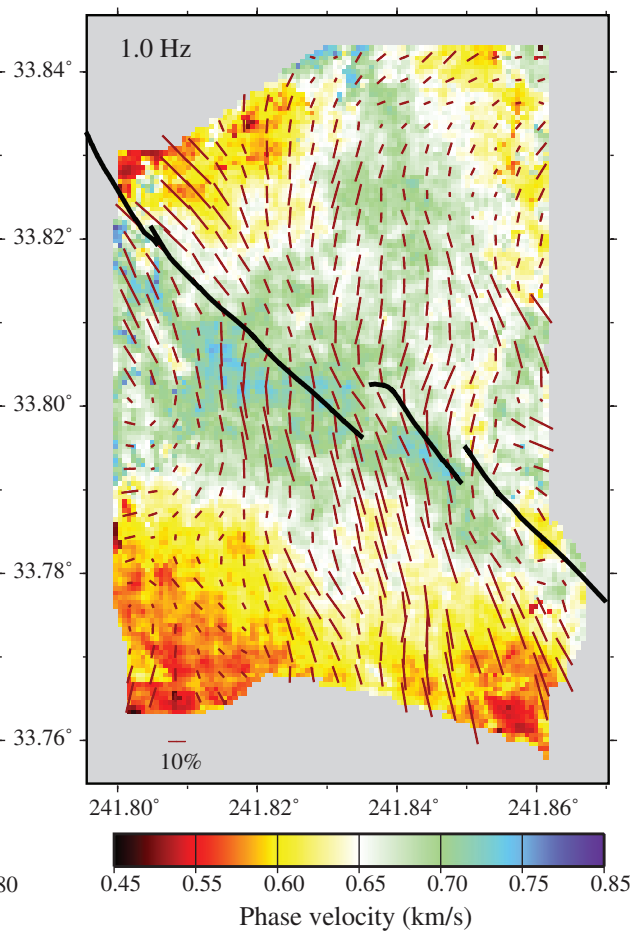

Figure 12. (a-c) Examples of $1.25-\mathrm{Hz}$ Rayleigh wave directionally dependent phase velocity measurements (red bars) for three locations shown in (d). The green dashed line in each plot shows the predicted directionally dependent phase velocities based on the best fitting anisotropy fast direction and amplitude. (d) The best-fitting $1.25-\mathrm{Hz}$ Rayleigh wave azimuthal anisotropy fast directions and amplitudes are summarized by the orientation and length of the red bars. The 1.25-Hz Rayleigh wave isotropic phase velocity map is shown in the background. (e) Same as (d) but for the 1.0-Hz Rayleigh wave.

a)

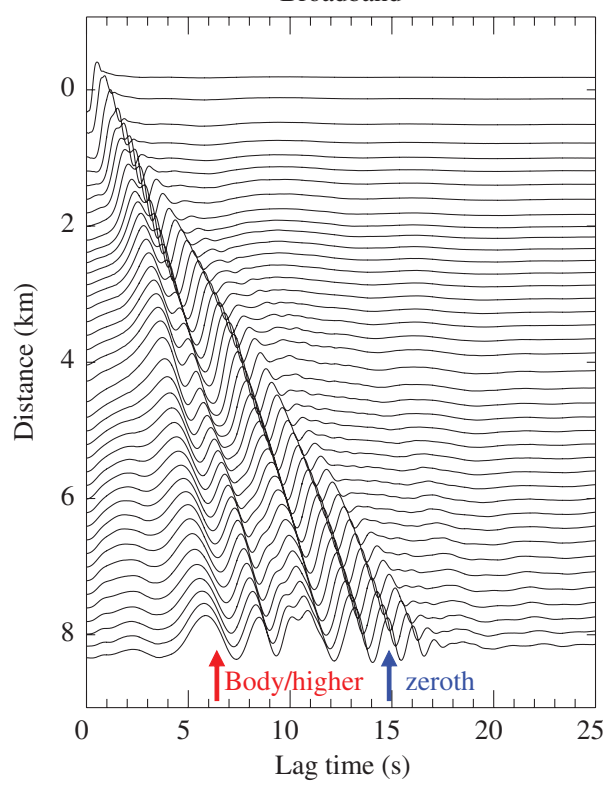

b)

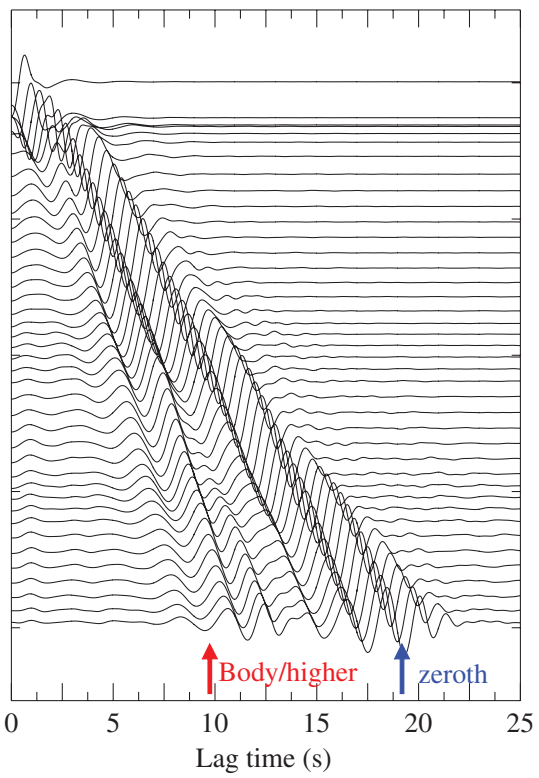

c)

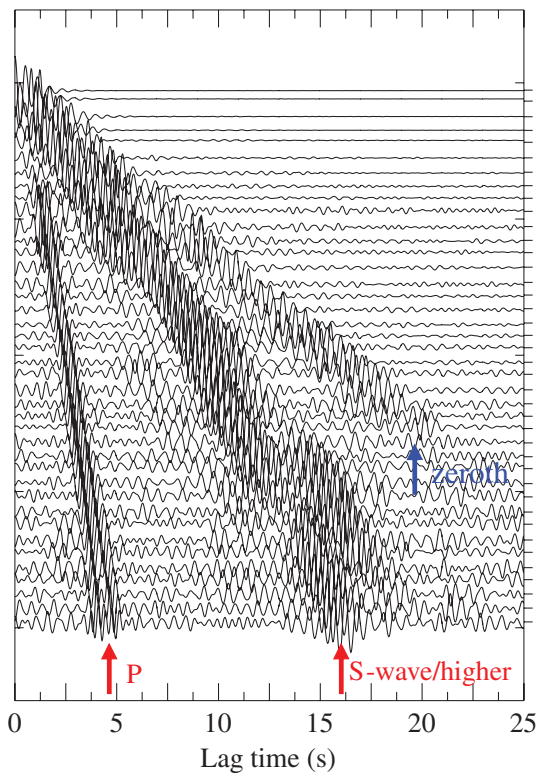

Figure 13. The record section of the stacked crosscorrelations, in which all crosscorrelations within each 200-m distance bin are stacked into one trace. (a-c) Without band-passed, $0.5-2-\mathrm{Hz}$ band-passed, and 2-4-Hz band-passed crosscorrelations, respectively. The fundamental Rayleigh wave signals and body wave/higher mode signals are indicated by blue and red arrows, respectively. 
Although using body waves to invert for structure is beyond the scope of this study, the ability to extract body wave signals from ambient noise shows an encouraging first step toward reflection imaging based on ambient noise.

\section{CONCLUSIONS}

In this study, we showed that ambient noise tomography can be applied to a dense industrial array to recover high-resolution shear velocity structure in the shallow crust. Although the array is designed to record active sources at high frequencies (up to $\sim 80 \mathrm{~Hz}$ ), low-frequency surface wave signals between 0.5 and $4 \mathrm{~Hz}$ can be extracted from ambient noise. The noise is likely oceanic below $1 \mathrm{~Hz}$ but is more scattered (homogeneous) above the $1-\mathrm{Hz}$ frequency. We show that by applying eikonal tomography, Rayleigh wave phase velocity maps can be determined and 3D shear velocity structure down to a $1-\mathrm{km}$ depth can be inverted for. The 3D model shows a clear correlation with the known geologic features, i.e., the shallow north-south velocity dichotomy across the Newport-Inglewood fault zone and the deeper fast-velocity anomaly associated with the fault zone itself. The ability to constrain a shallow structure based on ambient noise provides a cost-efficient way to complement traditional exploration seismology, considering that only passive signals are required. The $3 \mathrm{D}$ velocity model can also be used for hazard assessment and fault zone studies.

\section{ACKNOWLEDGMENTS}

The authors gratefully acknowledge NodalSeismic LLC and Signal Hill Petroleum, Inc., for permitting us to use the Long Beach data. We also thank K. Wapenaar, the associate editor, A. Verdel, and two anonymous reviewers for constructive comments and V. Tsai and C. Stork for helpful discussions.

\section{REFERENCES}

Barmin, M. P., M. H. Ritzwoller, and A. L. Levshin, 2001, A fast and reliable method for surface wave tomography: Pure and Applied Geophysics, 158 1351-1375, doi: 10.1007/PL00001225.

Bensen, G. D., M. H. Ritzwoller, M. P. Barmin, A. L. Levshin, F. Lin, M. P. Moschetti, N. M. Shapiro, and Y. Yang, 2007, Processing seismic ambient noise data to obtain reliable broad-band surface wave dispersion measurements: Geophysical Journal International, 169, 1239-1260, doi: 10.1111/j .1365-246X.2007.03374.x.

Coutant, O., 1996, Observation of shallow anisotropy on local earthquake records at the Garner Valley, Southern California, downhole array: Bulletin of the Seismological Society of America, 86, 477-488.

Crampin, S., 1994, The fracture criticality of crustal rocks: Geophysical Journal International, 118, 428-438, doi: 10.1111/j.1365-246X.1994 .tb03974.x.

de Lorenzo, S., and M. Trabace, 2011, Seismic anisotropy of the shallow crust in the Umbria-Marche (Italy) region: Physics of the Earth and Planetary Interiors, 189, 34-46, doi: 10.1016/j.pepi.2011.09.008.

Draganov, D., X. Campman, J. Thorbecke, A. Verdel, and K. Wapenaar, 2009, Reflection images from ambient seismic noise: Geophysics, $\mathbf{7 4}$ no. 5, A63-A67, doi: 10.1190/1.3193529.

Gouédard, P., H. Yao, F. Ernst, and R. D. van der Hilst, 2012, Surface wave eikonal tomography in heterogeneous media using exploration data: Geophysical Journal International, 191, 781-788, doi: 10.1111/j.1365246X.2012.05652.x.

Heidbach, O., M. Tingay, A. Barth, J. Reinecker, D. Kurfeß, and B. Müller, 2008, The World Stress Map database release 2008, accessed at http://dcapp3-14.gfz-potsdam.de/pub/stress_maps/stress_maps.html.

Herrmann, R. B., and C. J. Ammon, 2002, Computer programs in seismology: Surface waves, receiver functions and crustal structure: Saint Louis University.

Lin, F., M. P. Moschetti, and M. H. Ritzwoller, 2008, Surface wave tomography of the western United States from ambient seismic noise: Rayleigh and Love wave phase velocity maps: Geophysical Journal International, 173, 281-298, doi: 10.1111/j.1365-246X.2008.03720.x.

Lin, F., and M. H. Ritzwoller, 2011a, Helmholtz surface wave tomography for isotropic and azimuthally anisotropic structure: Geophysical Journal International, 186, 1104-1120, doi: 10.1111/j.1365-246X.2011 $.05070 . \mathrm{x}$.

Lin, F., and M. H. Ritzwoller, 2011b, Apparent anisotropy in inhomogeneous isotropic media: Geophysical Journal International, 186, 1205-1219, doi: 10.1111/j.1365-246X.2011.05100.x.

Lin, F., M. H. Ritzwoller, and R. Snieder, 2009, Eikonal tomography: Surface wave tomography by phase-front tracking across a regional broad-band seismic array: Geophysical Journal International, 177, 1091-1110, doi: 10.1111/j.1365-246X.2009.04105.x.

Lin, F., M. H. Ritzwoller, Y. Yang, M. P. Moschetti, and M. J. Fouch, 2011 Complex and variable crustal and uppermost mantle seismic anisotropy in the western United States: Nature Geoscience, 4, 55-61, doi: 10.1038/ ngeo1036.

Lin, F., B. Schmandt, and V. C. Tsai, 2012, Joint inversion of Rayleigh wave phase velocity and ellipticity using USArray: Constraining velocity and density structure in the upper crust: Geophysical Research Letters, 39, L12303, doi: 10.1029/2012GL052196.

Lin, F.-C., V. C. Tsai, B. Schmandt, Z. Duputel, and Z. Zhan, 2013 Extracting seismic core phases with array interferometry: Geophysical Research Letters, 40, doi: 10.1002/grl.50237.

Lobkis, O. I., and R. L. Weaver, 2001, On the emergence of the Green's function in the correlations of a diffuse field: Journal of the Acoustical Society of America, 110, 3011-3017, doi: 10.1121/1 1417528.

Moschetti, M. P., M. H. Ritzwoller, F. Lin, and Y. Yang, 2010, Crustal shear velocity structure of the western US inferred from ambient noise and earthquake data: Journal of Geophysical Research, 115, B10306, doi: 10.1029/2010JB007448

Poli, P., M. Campillo, and H. Pedersen, LAPNET Working Group, 2012b, Body-wave imaging of earth's mantle discontinuities from ambient seismic noise: Science, 338, 1063-1065, doi: 10.1126/science .1228194 .

Poli, P., H. A. Pedersen, and M. Campillo, The POLENET/LAPNET Working Group, 2012a, Emergence of body waves from cross-correlation of short period seismic noise: Geophysical Journal International, 188 549-558, doi: 10.1111/j.1365-246X.2011.05271.x.

Prieto, G. A., M. Denolle, J. F. Lawrence, and G. C. Beroza, 2011, On the amplitude information carried by ambient seismic field: Comptes Rendus Geoscience, 343, 600-614, doi: 10.1016/j.crte.2011.03.006.

Ruigrok, E., X. Campman, and K. Wapenaar, 2011, Extraction of P-wave reflections from microseisms: Comptes Rendus Geoscience, 343, 512525, doi: $10.1016 /$ j.crte.2011.02.006.

Sabra, K., P. Gersoft, P. Roux, and W. Kuperman, 2005, Extracting time-domain Green's function estimates from ambient seismic noise: Geophysical Research Letters, 32, L03310, doi: 10.1029/ 2004GL021862.

Saygin, E., and B. L. N. Kennett, 2012, Crustal structure of Australia from ambient seismic noise tomography: Journal of Geophysical Research, 117, B01304, doi: 10.1029/2011JB008403.

Shapiro, N. M., M. Campillo, L. Stehly, and M. H. Ritzwoller, 2005, High resolution surface wave tomography from ambient seismic noise: Science, 307, 1615-1618, doi: 10.1126/science. 1108339 .

Smith, M. L., and F. A. Dahlen, 1973, Azimuthal dependence of Love and Rayleigh-wave propagation in a slightly anisotropic medium: Journal of Geophysical Research, 78, 3321-3333, doi: 10.1029/ JB078i017p03321.

Smith, W. H. F., and P. Wessel, 1990, Gridding with continuous curvature splines in tension: Geophysics, 55, 293-305, doi: 10.1190/1.1442837.

Snieder, R., 2004, Extracting the Green's function from the correlation of coda waves: A derivation based on stationary phase: Physical Review E 69, 046610, doi: 10.1103/PhysRevE.69.046610.

Tsai, V. C., 2010, The relationship between noise correlation and the Green's function in the presence of degeneracy and the absence of equipartition Geophysical Journal International, 182, 1509-1514, doi: 10.1111/j.1365246X.2010.04693.x.

Wesnousky, S. G., 2005, The San Andreas and Walker Lane fault systems, western North America: Transpression, transtension, cumulative slip and the structural evolution of a major transform plate boundary: Journal of Structural Geology, 27, 1505-1512, doi: 10.1016/j.jsg.2005 .01 .015 .

Wielandt, E., 1993, Propagation and structural interpretation of non-plane waves: Geophysical Journal International, 113, 45-53, doi: 10.1111/j .1365-246X.1993.tb02527.x.

Wright, T., 1991, Structural geology and tectonic evolution of the Los Angeles Basin, in T. Wright, ed., Active Margin Basins: AAPG Memoir 52.

Yang, Y., M. H. Ritzwoller, and C. H. Jones, 2011a, Crustal structure determined from ambient noise tomography near the magmatic centers of 
the Coso region, southeastern California: Geochemistry, Geophysics, Geosystems, 12, Q02009, doi: 10.1029/2010GC003362.

Yang, Y., M. H. Ritzwoller, A. L. Levshin, and N. M. Shapiro, 2007 Ambient noise Rayleigh wave tomography across Europe: Geophysical Journal International, 168, 259-274, doi: 10.1111/j.1365-246X.2006 .03203.X.

Yang, Z., A. Sheehan, and P. Shearer, 2011b, Stress-induced upper crustal anisotropy in southern California: Journal of Geophysical Research, 116, B02302, doi: 10.1029/2010JB007655.

Yao, H., C. Beghein, and R. D. van der Hilst, 2008, Surface-wave array tomography in SE Tibet from ambient seismic noise and two-station analysis. II: Crustal and upper mantle structure: Geophysical Journal International, 173, 205-219, doi: 10.1111/j.1365-246X.2007.03696.x.

Yao, H., P. Gouedard, J. McGuire, J. Collins, and R. D. van der Hilst, 2011, Structure of young East Pacific Rise lithosphere from ambient noise cor- relation analysis of fundamental- and higher-mode Scholte-Rayleigh waves: Comptes Rendus Geoscience, 343, 571-583, doi: 10.1016/j.crte 2011.04.004

Yao, H., and R. D. van der Hilst, 2009, Analysis of ambient noise energy distribution and phase velocity bias in ambient noise tomography, with application to SE Tibet: Geophysical Journal International 179, 1113 1132, doi: 10.1111/j.1365-246X.2009.04329.x.

Yao, H. J., R. D. van der Hilst, and M. V. de Hoop, 2006, Surface-wave array tomography in SE Tibet from ambient seismic noise and two-station analysis. I: Phase velocity maps: Geophysical Journal International, 166, 732-744, doi: 10.1111/j.1365-246X.2006.03028.x.

Zhan, Z., S. Ni, D. Helmberger, and R. Clayton, 2010, Retrieval of Mohoreflected shear wave arrivals from ambient seismic noise: Geophysical Journal International, 182, 408-420, doi: 10.1111/j.1365-246X.2010 $.04625 . \mathrm{x}$ 